\title{
Early Respiratory Therapy Reduces Postoperative Atelectasis in Children Undergoing Lung Resection
}

\author{
Patrícia Nerys Kaminski, Luiz Alberto Forgiarini Junior PhD, \\ and Cristiano Feijó Andrade MD PhD
}

\begin{abstract}
BACKGROUND: Early physiotherapy reduces pulmonary complications after lung resection in adult patients. However, the effectiveness and the techniques used in postoperative physiotherapy in children undergoing lung resection have not been well described. Therefore, the standardization of a physiotherapeutic attendance after lung resection in children is necessary. METHODS: This was a retrospective and prospective, interventional, descriptive, and quantitative study. We evaluated 123 pediatric subjects undergoing lung resection. Fifty-two children were prospectively submitted to a standardized physiotherapy protocol that included a mask with a positive expiratory pressure of $10 \mathrm{~cm} \mathrm{H}_{2} \mathrm{O}$, expiratory rib cage compression, coughing, lifting the upper limbs, and ambulation, starting within the first 4 hours after surgery and continuing 3 times each day. A historical control group of $\mathbf{7 1}$ subjects received physiotherapeutic techniques without specific standardization and with variability in the start date and number of days attended. We recorded the presence of postoperative complications, prolonged air leak, postoperative bronchoscopy, the time of chest tube removal, and hospital stay following surgery. RESULTS: The group that received a standardized protocol of physiotherapy had fewer instances of atelectasis than the control group $(15.4 \%$ vs $7.6 \%, P=.01)$. Subjects in the control group were more likely than those in the intervention group to require fiberoptic bronchoscopy for bronchial toilet $(n=14[19.7 \%]$ vs $n=5$ [9.6\%], $P \leq .001)$. There was no difference in the time of drainage or hospital stay between the groups. CONCLUSIONS: Implementation of a standardized physiotherapeutic protocol after lung resection in children decreases atelectasis but does not reduce the time of chest tube removal or the duration of hospital stay. Key words: child; surgery; postoperative care; lung; physiotherapy; lung resection; positive expiratory pressure; pediatric; complications. [Respir Care 2013;58(5):805-809.

(C) 2013 Daedalus Enterprises]
\end{abstract}

\section{Introduction}

The effectiveness of and the techniques used in postoperative physiotherapy in children undergoing lung resection have not been well described. Early postoperative respiratory physiotherapy following lung resection reduces respiratory complications in adult patients. ${ }^{1,2}$ However,

\footnotetext{
Ms Kaminski and Dr Andrade are affiliated with the Department of Thoracic Surgery, Hospital da Criança Santo Antônio/Hospital de Clínicas de Porto Alegre, Porto Alegre, Rio Grande do Sul, Brazil. Dr Forgiarini is affiliated with the Department of Physiotherapy, Santa Casa Hospital Complex, Centro Universitário Metodista, Instituto Porto Alegre, Porto Alegre, Rio Grande do Sul, Brazil.
}

The authors have disclosed no conflicts of interest. children have specific physiological differences, suggesting that their postoperative complications may differ from those of adults. ${ }^{3,4}$

Children rarely undergo lung resection. ${ }^{5-8}$ The respiratory structures of children and adults differ in size, position, and function. ${ }^{9}$ The thoracic cage is highly compliant because the surrounding muscles remain immature and provide weak respiratory structural support. ${ }^{10-13}$ Further-

\footnotetext{
Correspondence: Cristiano Feijó Andrade MD PhD, Hospital da Criança Santo Antônio/Hospital de Clínicas de Porto Alegre, Avenida Independência 185, Porto Alegre, Rio Grande do Sul 90020-090 Brazil. E-mail: cristianofa@gmail.com.
}

DOI: $10.4187 /$ respcare. 01870 
more, the thoracic cage is formed by horizontal ribs that change the anteroposterior thoracic diameter during inspiration, resulting in a diaphragmatic overload. This is problematic because the diaphragm is the most important respiratory muscle. ${ }^{10-12}$

A variety of respiratory physiotherapeutic techniques have been evaluated for their ability to reduce postoperative pulmonary complications and previous pulmonary disease exacerbations. ${ }^{2,14,15}$ However, the details of many of these techniques have not been well described. ${ }^{14,15}$ In the literature there are few case reports mentioning the application of postoperative respiratory physiotherapy following lung resection in children. ${ }^{16-18}$ Additionally, none of these studies described an ideal time for beginning respiratory intervention maneuvers. Therefore, we proposed a standard protocol for early postoperative respiratory physiotherapy after lung resection in children.

To investigate whether establishing a predefined physiotherapeutic protocol can reduce postoperative complications, we compared a pediatric population of patients from a single institution who were submitted to lung resection during 2 different periods of time.

\section{Methods}

\section{Design}

This was a retrospective and prospective, interventional, descriptive, and quantitative study. From January 2005 to December 2008, we selected 127 consecutive pediatric patients who underwent lung resection at Santo Antônio Children's Hospital, Santa Casa Hospital Complex of Porto Alegre. A total of 136 lung resections were performed by the same surgeon (CFA). Seven subjects underwent 2 surgeries, and one subject underwent 3 surgeries at different times. Four subjects were excluded due to incomplete information on the physiotherapeutic approach. The remaining 123 subjects were divided into a retrospective group who did not receive standardized physiotherapy and a prospective group who underwent standardized physiotherapy protocol. Some of the subjects included in this study are described elsewhere. ${ }^{7}$ The study was approved by the Committee of Ethics and Research of Santa Casa Hospital Complex of Porto Alegre.

\section{Subjects}

One hundred twenty-three subjects were divided into a retrospective group (group 1), who received respiratory therapy of the physiotherapist's choosing, and a prospective group (group 2), in which a pre-established standard physiotherapeutic protocol was applied.

We retrospectively reviewed the charts of 71 subjects who did not receive standardized respiratory therapy in the

\section{QUICK LOOK}

\section{Current knowledge}

Positive airway pressure (PAP), secretion clearance techniques, and early ambulation are applied after upper abdominal and thoracic surgery to prevent atelectasis. The evidence that these techniques prevent postoperative pulmonary complications in adults is questionable, and the evidence in pediatric lung resection is sparse.

\section{What this paper contributes to our knowledge}

A pediatric, post-lung-resection protocol that included positive airway pressure, early ambulation, assisted cough, and arm raises decreased the incidence of atelectasis but did not reduce hospital stay or the time until chest tube removal.

postoperative period after lung resection (group 1). The subjects from group $2(n=52)$ were analyzed prospectively, following a standardized protocol of physiotherapy in the early postoperative period. The data analysis from group 1 was not performed until all the results from group 2 were collected. The analysis was carried out by a blinded researcher for the 2 groups. The subjects enrolled in group 1 underwent surgery between January 2005 and December 2006, and the subjects from group 2 underwent surgery between January 2007 and December 2008. The exclusion criteria for both groups included patients with incomplete medical records or who required mechanical ventilation more than 4 hours after surgery.

\section{Intervention}

The subjects in group 1 were subjected to various nonstandardized physical therapy techniques (mask positive expiratory pressure of $10 \mathrm{~cm} \mathrm{H}_{2} \mathrm{O}$, manual chest maneuvers, underwater seal, no standardization coughing, elevation of upper limbs) in which the start time and the number of days attended varied. The physical therapy protocol instituted in January 2007 consisted of 3 daily sessions, beginning within the first 4 hours after surgery and performed daily until the time of hospital discharge.

The care protocol consisted of the following physical therapy techniques: expiratory pressure of $10 \mathrm{~cm} \mathrm{H}_{2} \mathrm{O}$, expiratory rib cage compression, manually assisted coughing, lifting the upper limbs and ambulation, based on the patient's improvement. Each session consisted of $30 \mathrm{~min}$ of physiotherapy. All physiotherapists (5 members) were previously trained to standardize the different physiotherapeutic techniques. 


\section{Early Respiratory Therapy Reduces Postoperative Atelectasis}

The expiratory pressure device consisted of a face mask with a one-way valve for the adjustment of the expiratory resistance (5-20 $\left.\mathrm{cm} \mathrm{H}_{2} \mathrm{O}\right)$. The subject breathed in a tidal volume with a slightly active expiration, performing approximately 6-10 breaths for maintenance of a certain degree of lung volume, thus avoiding a full exhalation. ${ }^{19}$ In our protocol the subjects using the expiratory pressure mask performed 10 breaths 4 times, with intervals of $30 \mathrm{sec}-$ onds between the cycles. The value of $10 \mathrm{~cm} \mathrm{H}_{2} \mathrm{O}$ was chosen based on the literature, which recommends the use of values between 10 and $20 \mathrm{~cm} \mathrm{H}_{2} \mathrm{O}$ to resolve atelectasis. ${ }^{20-22}$ Based on our clinical practice, we observed that the use of $10 \mathrm{~cm} \mathrm{H}_{2} \mathrm{O}$ was possible for children younger than 4 years.

Expiratory rib cage compression consisted of a chest compression performed by the physiotherapist, with both hands placed over the chest wall. The compression was performed throughout the expiratory phase, with the goal of increasing the expiratory flow and displacing secretions from the large airways, for which the cough was more effective. ${ }^{23}$ The length of that maneuver depended on the perception of the physiotherapist regarding the clearance of secretions and the lung re-expansion ability of the subject. Nasopharyngeal aspiration was used for bronchial hygiene only in subjects who had no ability to remove secretions.

\section{Outcome Measures}

The end points for the 2 groups were postoperative pulmonary complications, postoperative bronchoscopy, the timing of chest tube removal, and hospital stay following surgery. Pulmonary complications included prolonged air leak, pneumothorax, atelectasis, and consolidation in the chest X-ray, which were evaluated by a team of radiologists blinded to the physiotherapeutic protocol. Prolonged air leak was defined as the escape of air through the chest tube lasting longer than 7 days. Postoperative flexible bronchoscopy was performed on subjects who presented with clinical and radiological worsening until the third postoperative day. The criteria that indicated bronchoscopy included the presence of copious secretions, diffuse bronchi, and persistent lung atelectasis and/or consolidation. The chest tubes were removed when the pleural fluid drainage was $\leq 3 \mathrm{~mL} / \mathrm{kg} / \mathrm{d}$, the lungs showed complete expansion in the chest $\mathrm{x}$-ray, and no air leaks were detected. The criteria for the subjects' discharge were at least 1 day after chest tube removal, absence of fever, and adequate lung expansion in the chest $\mathrm{x}$-ray.

\section{Data Analysis}

Statistical analyses were performed using statistics software (SPSS 14.0, SPSS, Chicago, Illinois). The categori-
Table 1. Characteristics of 123 Pediatric Subjects Undergoing Lung Resection

\begin{tabular}{cccc}
\hline \hline & Group 1 & Group 2 & $P$ \\
\hline Male, no. (\%) & $36(50.7)$ & $26(50)$ & .93 \\
Female, no. (\%) & $35(49.3)$ & $26(50)$ & \\
Age, mean \pm SD y & $6.83 \pm 4.83$ & $7 \pm 5.44$ & .85 \\
\hline
\end{tabular}

Table 2. Postoperative Diagnoses of 123 Pediatric Lung Resection Subjects

\begin{tabular}{lccc}
\hline \hline & $\begin{array}{c}\text { Group 1 } \\
(n=71) \\
\text { no. }(\%)\end{array}$ & $\begin{array}{c}\text { Group 2 } \\
(n=52) \\
\text { no. }(\%)\end{array}$ & $P$ \\
\hline Infectious disease* & $38(53.5)$ & $36(69.2)$ & .98 \\
Metastasis/tumor & $17(23.9)$ & $8(15.4)$ & .36 \\
Congenital lung malformation $\dagger$ & $14(19.7)$ & $5(9.6)$ & .59 \\
Other & $2(2.8)$ & $3(5.8)$ & .72 \\
& & & \\
$*$ Bronchiectasis, tuberculosis, sequelae of bronchiolitis obliterans, necrotizing pneumonia. \\
$\dagger$ Congenital cystic adenomatoid malformation, lung sequestration, congenital lobar \\
emphysema. \\
\end{tabular}

Table 3. Types of Lung Resection

\begin{tabular}{lccc}
\hline \hline & $\begin{array}{c}\text { Group } 1 \\
\text { no. }\end{array}$ & $\begin{array}{c}\text { Group } 2 \\
\text { no. }\end{array}$ & $P$ \\
\hline Segmentectomy & 41 & 29 & .82 \\
Lobectomy & 20 & 21 & .16 \\
Pneumectomy & 2 & 2 & .75 \\
Lobectomy + segmentectomy & 8 & 10 & .23 \\
Bilobectomy + segmentectomy & 1 & 1 & .81 \\
Bilobectomy & 1 & 0 & .41 \\
\hline
\end{tabular}

cal variables are described by the frequency, whereas continuous variables are expressed as the mean and standard deviation. The Kolmogorov-Smirnov test was used to assess the normality of the variables. For the analysis of the variables, we used the Fisher exact test, the chi-square test, and the Student $t$ test, with a significance level of 5\% $(P<.05)$.

\section{Results}

The characteristics for both groups prior to surgery are shown in Table 1. The main postoperative pathological findings in both groups are described in Table 2, and the types of lung resections performed are described in Table 3.

Twenty-two subjects (30.9\%) in group 1 had postoperative complications (Table 4), which mainly consisted of atelectasis, pneumothorax, and prolonged air leak. In group 2, only 9 subjects developed pulmonary complica- 
Table 4. Postoperative Complications After Lung Resection

\begin{tabular}{lclc}
\hline \hline & $\begin{array}{c}\text { Group 1 } \\
\text { no. (\%) }\end{array}$ & $\begin{array}{l}\text { Group 2 } \\
\text { no. (\%) }\end{array}$ & $P$ \\
\hline Complications & $22(30.9)$ & $9(17.3)$ & .01 \\
$\quad$ Atelectasis & $11(15.5)$ & $4(7.7)$ & .01 \\
Pneumothorax & $5(7.0)$ & $4(7.7)$ & .89 \\
Prolonged air leakage & $3(4.2)$ & $1(1.9)$ & .68 \\
Other* & $12(16.9)$ & $1(1.9)$ & .01 \\
2 Complications & $7(9.9)$ & $1(1.9)$ & .08 \\
3 Complications & $1(1.4)$ & 0 & .81
\end{tabular}

* Chylothorax, empyema, subcutaneous emphysema, pleural effusion, pulmonary necrosis.

tions during the postoperative period $(17.3 \%, P \leq .001)$. The need for fiberoptic bronchoscopy for bronchial toilet was higher in the control group $(n=14,19.7 \%)$ compared to the intervention group $(n=5,9.6 \%, P \leq .001)$.

There was no significant difference in the timing of chest tube removal $(2.71 \pm 2.15 \mathrm{~d}$ vs $3.28 \pm 2.65 \mathrm{~d})$ or hospital stay after surgery $(9.9 \pm 9.1 \mathrm{~d}$ vs $8.48 \pm 5.21 \mathrm{~d})$ between groups 1 and 2 .

\section{Discussion}

This report is the first in the literature to specifically study and describe the use and the effectiveness of physiotherapy in a pediatric population submitted to lung resection. We showed that the establishment of a standard physiotherapy protocol is beneficial in this age group and can reduce atelectasis.

Adult patients undergoing lung resection are at a high risk for developing postoperative pulmonary complications with serious consequences. The main postoperative respiratory complications after lung resection include pneumonia, pulmonary infiltration, atelectasis, and respiratory failure, which often occur 2 to 3 days after surgery. ${ }^{24,25}$ Although children can present respiratory complications that are as serious as those of adults, adult patients often present comorbidities that significantly increase their risk for pulmonary complications and increase the postoperative morbidity and mortality. ${ }^{25,26}$ Infants undergoing surgical procedures involving the chest may show reduced oxygen saturation prior to and after surgery as a result of changes in thoracic and lung compliance. ${ }^{27}$ Although we did not observe life-threatening complications in our series, postoperative complications were reduced with the standardized physiotherapy protocol.

The postoperative period following thoracotomy results in a decrease in the functional residual capacity and an increase in the final closing volume, which may lead to atelectasis. ${ }^{28}$ In children, the chest wall offers less resistance to the retraction forces of the lungs, causing a shorter volume at the end of expiration and facilitating airway collapse. ${ }^{29,30}$ Moreover, the postoperative reduction of ciliary motion and expectoration promote sputum retention and facilitate atelectasis. ${ }^{26}$ Our findings corroborate this observation, as atelectasis was the most frequent pulmonary complication. Respiratory complications were reduced in the subjects in group 2, who received more attention and care during the physiotherapy session.

Despite conflicting results regarding the benefits of postoperative respiratory therapy following thoracic surgical procedures, ${ }^{31,32}$ Varela et al found that lobectomy patients showed a reduction in pulmonary atelectasis without increasing the costs of the procedure. ${ }^{15}$ Although an earlier study reported no reduction in postoperative complications, the authors encouraged the use of routine respiratory therapy and emphasized the importance of a multidisciplinary team for the postoperative care of surgical patients. ${ }^{31}$ Gunn and Goding showed that professional training plays a role in the clinical practice of physiotherapists working in a community National Health Service setting. Training significantly improves both confidence and competence, enabling individuals to form effective therapeutic relationships with patients and other members of their teams. ${ }^{33}$

Training pediatric physiotherapists is time-consuming, due to variations in how the maneuvers are performed and applied. The professionals who provided the physical therapy intervention in this study had been previously trained to apply specific techniques. The selection of the maneuvers was determined by the standard protocol. Additionally, physiotherapy for pediatric populations has some unique features because it requires an interaction between the therapist and the patient, and school-age children cannot always understand and cooperate with the therapeutic techniques. ${ }^{4}$ Most of our subjects were younger than 7 years of age, and the success of the physiotherapy mainly depended on the physiotherapist. We did not observe any contraindications or collateral effects using this physiotherapeutic protocol. Our study shows that standardizing the physiotherapy protocol in terms of the number of sessions, the types of maneuvers, and the time that physical therapy is initiated reduces pulmonary complications.

The design of this study has certain limitations that should be considered. First, we compared a historical group with a prospective group, which could result in a tendency to favor the prospective group. We attempted to reduce this bias by analyzing both groups at the end of the data collection phase. Additionally, all subjects in this study were operated on by the same surgeon with the same surgical technique, and the postoperative physiotherapy was performed by the same group, thus avoiding the possibility of interference in the final results. Although a more appropriate study to assess a new training protocol would be a randomized clinical trial that compares 2 groups with 


\section{Early Respiratory Therapy Reduces Postoperative Atelectasis}

and without intervention, the exclusion of postoperative physiotherapy could be detrimental to such a group of patients. We hope to stimulate future studies on respiratory physiotherapy involving children undergoing lung resection or other surgical procedures.

\section{Conclusions}

The implementation of a standardized physiotherapeutic protocol after lung resection in children decreases atelectasis but does not reduce hospital stay or the time to chest tube removal.

\section{REFERENCES}

1. Pehlivan E, Turna A, Gurses A, Gurses HN. The effects of preoperative short-term intense physical therapy in lung cancer patients: a randomized controlled trial. Ann Thorac Cardiovasc Surg 2011;17(5): 461-468.

2. Reeve J, Denehy L, Stiller K. The physiotherapy management of patients undergoing thoracic surgery: a survey of current practice in Australia and New Zealand. Physiother Res Int 2007;12(2):59-71.

3. Doyle RL. Assessing and modifying the risk of postoperative pulmonary complications. Chest 1999;115(5 Suppl):77S-81S.

4. Oberwaldner B. Physiotherapy for airway clearance in paediatrics. Eur Respir J 2000;15(1):196-204.

5. Ayed AK, Al-Rowayeh A. Lung resection in children for infectious pulmonary diseases. Pediatr Surg Int 2005;21(8):604-608.

6. Andrade CF, Ferreira HP, Fischer GB. Congenital lung malformations. J Bras Pneumol 2011;37(2):259-271.

7. Ferreira HP, Fischer GB, Felicetti JC, Camargo Jde J, Andrade CF. [Surgical treatment of congenital lung malformations in pediatric patients]. J Bras Pneumol 2010;36(2):175-180. Article in Portuguese.

8. Kanngiesser P, Liewald F, Halter G, Sunder-Plassmann L. Thoracic surgery in children. Eur J Cardiothorac Surg 2005;28(1):50-55.

9. Graeber GM, Nazim M. The anatomy of the ribs and the sternum and their relationship to chest wall structure and function. Thorac Surg Clin 2007;17(4):473-489.

10. Carlo WA, Martin RJ. Principles of neonatal assisted ventilation. Pediatr Clin N Am 1986;33(1):221-237.

11. Dezateux C, Stocks J. Lung development and early origins of childhood respiratory illness. Br Med Bull 1997;53(1):40-57.

12. Greenough A, Roberton NR. Neonatal ventilation. Early Hum Develop 1986;13(2):127-136.

13. Allen J, Gripp KW. Development of the thoracic cage. In: Haddad GG, Abman SH, Chernick V, editors. Mechanisms of pediatric respiratory disease. Hamilton, Ontario: BC Decker; 2002:124-138.

14. Kigin CM. Chest physical therapy for the postoperative or traumatic injury patient. Phys Ther 1981;61(12):1724-1736.

15. Varela G, Ballesteros E, Jimenez MF, Novoa N, Aranda JL. Costeffectiveness analysis of prophylactic respiratory physiotherapy in pulmonary lobectomy. Eur J Cardiothorac Surg 2006;29(2):216-220.

16. Otgun I, Karnak I, Tanyel FC, Senocak ME, Buyukpamukcu N. Surgical treatment of bronchiectasis in children. J Pediatr Surg 2004; 39(10): 1532-1536.
17. Blyth DF, Buckels NJ, Sewsunker R, Soni MA. Pneumonectomy in children. Eur J Cardiothorac Surg 2002;22(4):587-594.

18. Zhang P, Jiang G, Ding J, Zhou X, Gao W. Surgical treatment of bronchiectasis: a retrospective analysis of 790 patients. Ann Thorac Surg 2010;90(1):246-250.

19. Pryor J, Webber B. Fisioterapia para Problemas Respiratórios e Cardíacos. Rio de Janeiro: Guanabara Koogan; 2002. Book in Portuguese.

20. Forshag MS, Cooper AD, Jr. Postoperative care of the thoracotomy patient. Clin Chest Med 1992;13(1):33-45.

21. Martin RJ, Rogers RM, Gray BA. Mechanical aids to lung expansion. The physiologic basis for the use of mechanical aids to lung expansion. Am Rev Respir Dis 1980;122(5 Pt 2):105-107.

22. Postiaux G. Fisioterapia respiratória pediátrica: o tratamento guiado por ausculta pulmonar. Porto Alegre: Artmed; 2004. Book in Portuguese.

23. Soares PRD, Dias AS, Jardim JRB. Fisioterapia respiratória para o paciente pneumológico. In: Silva LCC, editor. Condutas em pneumologia. Vol. II. Rio de Janeiro: Revinter; 2001:903-916. Book in Portuguese.

24. Korst RJ, Humphrey CB. Complete lobar collapse following pulmonary lobectomy. Its incidence, predisposing factors, and clinical ramifications. Chest 1997;111(5):1285-1289.

25. Sanchez PG, Vendrame GS, Madke GR, Pilla ES, Camargo Jde J, Andrade $\mathrm{CF}$, et al. Lobectomy for treating bronchial carcinoma: analysis of comorbidities and their impact on postoperative morbidity and mortality. J Bras Pneumol 2006;32(6):495-504.

26. Stephan F, Boucheseiche S, Hollande J, Flahault A, Cheffi A, Bazelly B, et al. Pulmonary complications following lung resection: a comprehensive analysis of incidence and possible risk factors. Chest 2000;118(5):1263-1270.

27. Mattioli G, Buffa P, Granata C, Fratino G, Rossi G, Ivani G, et al. Lung resection in pediatric patients. Pediatr Surg Int 1998;13(1):1013.

28. Sabanathan S, Eng J, Mearns AJ. Alterations in respiratory mechanics following thoracotomy. J R Coll Surg Edinb 1990;35(3):144-150.

29. American Thoracic Society/European Respiratory Society. Respiratory mechanics in infants: physiologic evaluation in health and disease. Am Rev Respir Dis 1993;147(2):474-496.

30. Motoyama EK, Yang CI, Deeney VF. Thoracic malformation with early-onset scoliosis: effect of serial VEPTR expansion thoracoplasty on lung growth and function in children. Paediatr Respir Rev 2009; 10(1):12-17.

31. Arcêncio L, Souza MD, Bortolin BS, Fernandes AC, Rodrigues AJ, Evora PR. Pre-and postoperative care in cardiothoracic surgery: a physiotherapeutic approach. Rev Bras Cir Cardiovasc 2008;23(3): 400-410.

32. O'Donohue WJ Jr. National survey of the usage of lung expansion modalities for the prevention and treatment of postoperative atelectasis following abdominal and thoracic surgery. Chest 1985;87(1): 76-80.

33. Gunn H, Goding L. Continuing professional development of physiotherapists based in community primary care trusts: a qualitative study investigating perceptions, experiences and outcomes. Physiotherapy 2009;95(3):210-215. 